Artículo científico

Volumen 31(3):635-647. Septiembre-diciembre, 2020

e-ISSN 2215-3608, doi:10.15517/am.v31i3.39940

http://www.revistas.ucr.ac.cr/index.php/agromeso

\title{
Diagnóstico financiero y biofísico para la producción de semilla de papa ${ }^{1}$
}

\section{Financial and biophysical analysis for the potato seed production}

\author{
S.C.Insuasty-Córdoba², H.S. Ramos-Zambrano², C.A. Marcillo-Paguay ${ }^{2}$, H.V. López-Peñafiel2 ${ }^{2}$, J.F. Mateus- \\ Rodríguez, E. Martínez-Pachón ${ }^{2}$
}

1 Recepción: 4 de diciembre, 2019. Aceptación: 2 de abril, 2020. Investigación presentada como parte del macroproyecto "Mejoramiento tecnológico y productivo del sistema papa en Nariño", financiado por el Fondo de Ciencia Tecnología e Innovación del Sistema General de Regalías (SGR), ejecutado por la Gobernación de Nariño bajo acuerdo tripartita 480-15 con los operadores Corporación Colombiana de Investigación Agropecuaria (Agrosavia) y Universidad de Nariño, que se llevo a cambo en el año 2017 y 2018.

2 Corporación Colombiana de Investigación Agropecuaria (AGROSAVIA). Centro de Investigación Obonuco, kilómetro 5, vía Pasto - Obonuco, 520001, Nariño, Colombia. sinsuasty@agrosavia.co (autor para correspondencia; https://orcid.org/0000-0003-3120-5159); hramos@agrosavia.co (https://orcid.org/0000-0001-7959-9834); cmarcillo@agrosavia.co（https://orcid.org/0000-0001-9100-2463); hvlopez@agrosavia.co (https://orcid.org/0000-0002-3504-0761); emartinezp@ agrosavia.co (https://orcid: 000-0002-5144-132X).

3 Corporación Colombiana de Investigación Agropecuaria (AGROSAVIA). CI Palmira, Carrera 36A con Calle 23, 763531, Palmira, Valle del Cauca, Colombia.jmateus@agrosavia.co (https://orcid.org/0000-0002-5516-7604).

\section{Resumen}

Introducción. En Colombia se han realizado esfuerzos por masificar el uso de semilla certificada, sin embargo, el desconocimiento de los productores en sus ventajas, la inexistencia de una oferta estable y la dependencia al precio de papa comercial, limitan la sostenibilidad de su producción. Objetivo. Evaluar financiera y biofísicamente la producción de semilla de papa en el departamento de Nariño, (Colombia). Materiales y Métodos. Se evaluó el uso, evolución, adopción y costos de semilla, entre mayo de 2016 y abril de 2018, para consolidar bases que permitan crear modelos de producción sostenibles. Dicha información fue recopilada por encuestas a 1018 hogares productores en veintiún municipios de la localidad. Para definir los parámetros biofísicos se utilizó una entrevista semiestructurada para extraer rangos y aptitudes en capas cartográficas, que se enmarcaron en un área con precipitación entre 800 a $1600 \mathrm{~mm}$ año ${ }^{-1}$, suelos francos con pendientes del $15 \%$, altitud entre 2800 a $3200 \mathrm{msnm}$ y temperatura de 8 a 15 ${ }^{\circ} \mathrm{C}$. El comportamiento del agronegocio se estableció con proyección de costos de producción a siete años, a partir de revisión de fuentes primarias y cifras de áreas sembradas durante los últimos diez semestres, que fue cotejada con la estimación del porcentaje de uso actual de semilla en la región para establecer la demanda potencial. Resultados. La carga económica no permitió generar rendimientos significativos en una sola hectárea $(\overline{\mathrm{X}}: 6 \%)$, porque fueron inferiores al de la producción de papa comercial ( $\overline{\mathrm{x}}: 18 \%$ ), con un promedio $20 \%$ y una tasa interna de retorno (TIR) del $28 \%$. Conclusión. El área mínima para superar la rentabilidad de la producción de papa comercial fue de tres hectáreas, logrando suministrar el $3 \%$ de la demanda de semilla. Las zonas con mayor potencial para producción fueron los Municipios de Pasto, Tangua y Sapuyes.

Palabras claves: semilla certificada, zona de aprovechamiento, oferta demanda.

\footnotetext{
Abstract

Introduction. Efforts have been made in Colombia to increase the use of certified seed. However, the lack of knowledge among producers about its advantages, the absence of a stable supply and the dependence on commercial 
potato prices, limit the sustainability of its production. Objective. To evaluate financially and biophysically the production of seed potatoes in the department of Nariño, Colombia. Materials and methods. The use, evolution, adoption, and costs of potato seed were evaluated between May 2016 and April 2018, to consolidate bases that allow creating sustainable production models. This information was collected through surveys to 1018 producing households in twenty-one local municipalities. To define the biophysical parameters, a semi-structured interview was used to extract ranges and aptitudes in cartographic layers, which were framed in an area with rainfall between 800 to $1600 \mathrm{~mm}$ year ${ }^{-1}$, loamy soils with slopes of $15 \%$, altitude between 2800 to 3200 m.a.s.l. and temperature of 8 to $15^{\circ}$ $\mathrm{C}$. The agribusiness behavior was established with a seven-year projection of production costs, based on a review of primary sources and figures of areas sown during the last ten semesters, which was compared with the estimate of the percentage of current seed use in the region to establish the potential demand. Results. The economic burden did not allow generating significant yields in a single hectare $(\overline{\mathrm{x}}: 6 \%)$, because they were lower than the commercial potato production ( $\overline{\mathrm{X}}: 18 \%$ ), with an average of $20 \%$ and internal rate of return (IRR) of $28 \%$. Conclusion. The minimum area to overcome the profitability of commercial potato production was three hectares, managing to supply $3 \%$ of the seed demand. The areas with greatest potential for production were the municipalities of Pasto, Tangua, and Sapuyes.

Keywords: certified seed, harvesting area, supply, demand.

\section{Introducción}

La papa (Solanum tuberosum) constituye el cuarto alimento de mayor consumo en el mundo (Borba, 2008). Sus niveles considerables de nutrientes y su capacidad adaptativa para diferentes pisos térmicos han hecho que se catalogue como un alimento con alta expansión en cuanto a su producción en países desarrollados, y que sea especialmente reconocido en regiones como China e India, que presentan índices demográficos altos.

El principal problema en la producción de la papa ha sido la calidad de la semilla (Esprella et al., 2012), que dificulta generar acuerdos comerciales. Este panorama es compartido en América Latina, ya que los productores de papa, en especial en países en vías de desarrollo, no utilizan semilla certificada (Ezeta, 2001), a excepción de Chile, Argentina y México, en razón a la baja tasa de multiplicación vegetativa de la papa, la debilidad de las asociaciones de productores y la escasa coordinación entre la oferta y la demanda para la producción de semilla.

Si bien se destaca el alto potencial que tiene la región Andina en la producción del tubérculo (Devaux et al., 2010), esto también sugiere la necesidad de implementar políticas que fomenten la investigación y la innovación en procesos de articulación pública y privada para el desarrollo de la cadena a nivel local e internacional. Bajo este contexto, desde el 2011 se han realizado grandes esfuerzos respecto a la cadena agroalimentaria de la papa en Colombia para masificar el uso de semillas certificadas. Según el informe de gestión del Instituto Colombiano Agropecuario de 2017 (ICA, 2018), en Colombia se produjeron $13585892 \mathrm{~kg}$ de semilla certificada que se sembraron en 9057 ha correspondientes al 6,8\% del área total sembrada, que para 2017 fue de 132161 ha, de acuerdo con los datos presentados para la cadena de papa en 2018 (MADR, 2018). Este bajo uso es generado por el aparente alto costo de la semilla y la incertidumbre que esta relación genera entre la oferta y la demanda.

La mayor parte de los productores de papa desconocen las ventajas técnicas y la necesidad económica de la adopción de la semilla certificada, la inexistencia de una oferta estable en todas las zonas productoras y, fundamentalmente, el hecho de la dependencia de su precio respecto al de la papa comercial.

El objetivo del estudio fue la evaluación financiera y biofísica de la producción de semilla de papa en el departamento de Nariño (Colombia). 


\section{Materiales y métodos}

\section{Área de estudio}

El estudio se llevó a cabo entre mayo de 2016 y abril de 2018 en veintiún municipios productores de papa (Solanum tuberosum L.), localizados en el suroccidente de los Andes de Colombia (departamento de Nariño), entre los $0^{\circ} 39^{\prime} 37,8^{\prime \prime}$ y $1^{\circ} 14^{\prime} 4,49^{\prime \prime}$ de latitud norte y $\operatorname{los} 77^{\circ} 51^{\prime} 9,68$ " y $77^{\circ} 7$ ' 53 " longitud oeste. Las áreas están agrupadas en tres subregiones: Centro (conformada por los municipios de Pasto, Tangua y Yacuanquer), Exprovincia de Obando (Cumbal, Ipiales, Guachucal, Pupiales, Córdoba, Cuaspud, Gualmatán, Puerres, Aldana, Potosí, Contadero e Iles) y Sabana (Túquerres, Ospina, Sapuyes, Imués, Guaitarilla y Providencia).

\section{Categorías de semilla}

Para el análisis de costos fue necesario tomar la categorización de semillas formales establecidas por el ICA (2015) en la Resolución 3168 del 7 de septiembre del 2015, así:

- Categoría super-élite: esquejes, plántulas in vitro y minitubérculos.

- Categoría élite: minitubérculos.

- Categoría básica: resulta de la multiplicación de élite.

- Categoría registrada: descendiente de básicas.

- Categoría certificada: descendiente de básicas o registradas.

- En todas las categorías se admiten hasta dos generaciones que deben ser inscritas.

\section{Variedad de estudio}

Diacol Capiro se conoce en el mercado con el nombre alterno de "R-12". Presenta porte medio, follaje verde oscuro, floración media y muy poca formación de frutos. Presenta alta adaptabilidad (entre 1800 y $3200 \mathrm{msnm}$ ) y se considera una variedad relativamente tardía (165 días, a $2600 \mathrm{msnm}$ ). Es la principal variedad para procesamiento en Colombia, tanto en hojuela como en bastón, por presentar un color de carne crema claro y buena respuesta a la fritura. Su rendimiento en condiciones óptimas de cultivo es superior a las $40 \mathrm{t} \mathrm{ha}^{-1} \mathrm{y}$ su periodo de reposo es de tres meses $\left(15^{\circ} \mathrm{C}\right.$ y $75 \%$ H.R.). Se cultiva en todas las regiones del país (Ñústez, 2011).

\section{Diseño estadístico}

Con base en el número total de productores de papa (16 861), reportados en el Consolidado Agropecuario publicado por la Secretaria de Agricultura de la Gobernación de Nariño para el año 2013 (Gobernación de Nariño, 2013), y a partir de la fórmula propuesta por Murray y Larry (2009), con un nivel de confianza del $98 \%$ y un margen de error del 2\%, se estableció para el año 2016 una muestra representativa de 1018 hogares en el área de estudio. La encuesta, sobre dimensiones de bienestar y manejo del sistema productivo a partir de trescientas preguntas tipo única repuesta, de selección múltiple y abiertas, que incluía interrogantes sobre la producción de semilla, se aplicó entre mayo y septiembre de 2016, donde se obtuvo información de las variedades. Diacol Capiro, Pastusa Suprema, Unica, Criolla Colombia, Parda Pastusa, Betina, Morasurco, Roja Nariño, Mambera y Esmeralda, descritas en las variedades colombianas de papa (Ñustez, 2011).

Para la delimitación de agroecosistemas para producción de semilla según características biofísicas, se realizaron entrevistas semiestructuras (Díaz-Bravo et al., 2013) a productores y organizaciones dedicadas a esta 
actividad. Como resultado, se obtuvieron las características biofísicas de altitud, temperatura y textura de suelo, con las cuales se extrajeron rangos y aptitudes en cada capa cartográfica para realizar una superposición de estas. Para la delimitación del área potencial para la producción de semilla de papa se utilizaron los mapas temáticos de las características biofísicas antes mencionadas, junto con el área de influencia de los puntos tomados en la encuesta (ubicación de lotes), que se consideraron como áreas de producción comercial para eliminarlas posteriormente y delimitar las áreas donde se podría producir semilla sin tener presión de lotes de producción de papa a una distancia mínima de $2000 \mathrm{~m}$.

Para determinar el comportamiento del negocio de producción de semilla de papa de calidad, se realizó una proyección de costos de producción a siete años, como lapso de tiempo mínimo para recuperar la inversión (Morales y Morales, 2014), a partir de la revisión de fuentes primarias (entrevistas semiestructuradas) y fuentes secundarias que indican el comportamiento de los precios en los últimos cinco años según el Sistema de Información de Precios (SIPSA) del Departamento Administrativo Nacional de Estadística (DANE, 2018a) y la Federación Colombiana de Productores de Papa (Fedepapa, 2018). Los supuestos financieros consideraron: ingresos brutos correspondiente a la venta de papa variedad Diacol Capiro de acuerdo a rendimientos de producción; 23,3\% para papa de primera / muy grande ( $>90 \mathrm{~mm}$ ) (precio de venta, USD: $\$ 11,10), 69,8 \%$ para segunda/mediana $(31-90 \mathrm{~mm}$ ) (precio de venta, USD: $\$ 20,3)$ y $6,9 \%$ para tercera/muy pequeña $(<30 \mathrm{~mm}$ ) (precio de venta, USD: $\$ 5,5)$; una tasa de oportunidad, establecida a partir de una tasa promedio DTF (depósito a término fijo) para el año 2017 del 4,50 \% (BANREP, 2019), tasa esperada de inflación de 3,2 \% para 2018 (DANE, 2018b), y bajo criterios de los investigadores de acuerdo al contexto económico de la región, una tasa esperada para el inversionista del 5,5\% y una para el empresario del $5 \%$ (Cámara de Comercio de Pasto, 2017), parámetros requeridos en el cálculo de la tasa interna de oportunidad (ecuación 1), que se constituyeron en comparativo entre los indicadores valor presente neto o actual (VPN/VAN) (Puente-Montañez, 2011); tasa interna de retorno (TIR) (Mete, 2014); periodo de recuperación de la inversión (PRI) y relación costo beneficio (B/C) (Gittinger, 1983; Cubbage et al., 2011). En el caso de la TIR y el VPN, los supuestos se establecieron en considerar que los ingresos serán reinvertidos a una tasa de expectativa o TIR hasta el final del emprendimiento y que los egresos tendrán un costo financiero igual a la tasa de expectativa o TIR (Mete, 2014).

$\mathrm{TIO}=\mathrm{DTFP}_{90 \text { días }}-\pi_{\mathrm{e}}+\mathrm{TEI}+\mathrm{TEE} \quad$ (ecuación 1$)$

Donde:

TIO: tasa interna de oportunidad.

DTFP: depósito a término fijo de 90 días, promedio 2017.

$\pi_{\mathrm{e}}$ : inflación esperada 2018.

TEI: tasa esperada por el inversionista.

TEE: tasa esperada por el empresario.

Los cálculos monetarios fueron establecidos a partir del comportamiento promedio del dólar en Colombia para el 2017 (1 USD = 2951 COP) y de las cifras de áreas sembradas en el departamento de Nariño durante los últimos ocho años, junto con la estimación del porcentaje de uso actual de semilla certificada en la región, con lo cual se estableció la demanda potencial de semilla de calidad en Nariño y se hizo una proyección de costos y rentabilidad bajo los escenarios de cosecha de una a siete hectáreas cultivadas. 


\section{Resultados}

\section{Uso de semilla de papa a nivel departamental}

Para el departamento de Nariño, con base a los datos obtenidos en la encuesta, se determinó que los productores de los veintiún municipios requirieron para la siembra de papa en 2359 ha un total de 3299 t, distribuidas entre un $9,85 \%$ de semilla certificada (325 t), 65,54\% de semilla propia (2162 t) y 24,61\% descendiente de semilla certificada (812 t) (Figura 1).

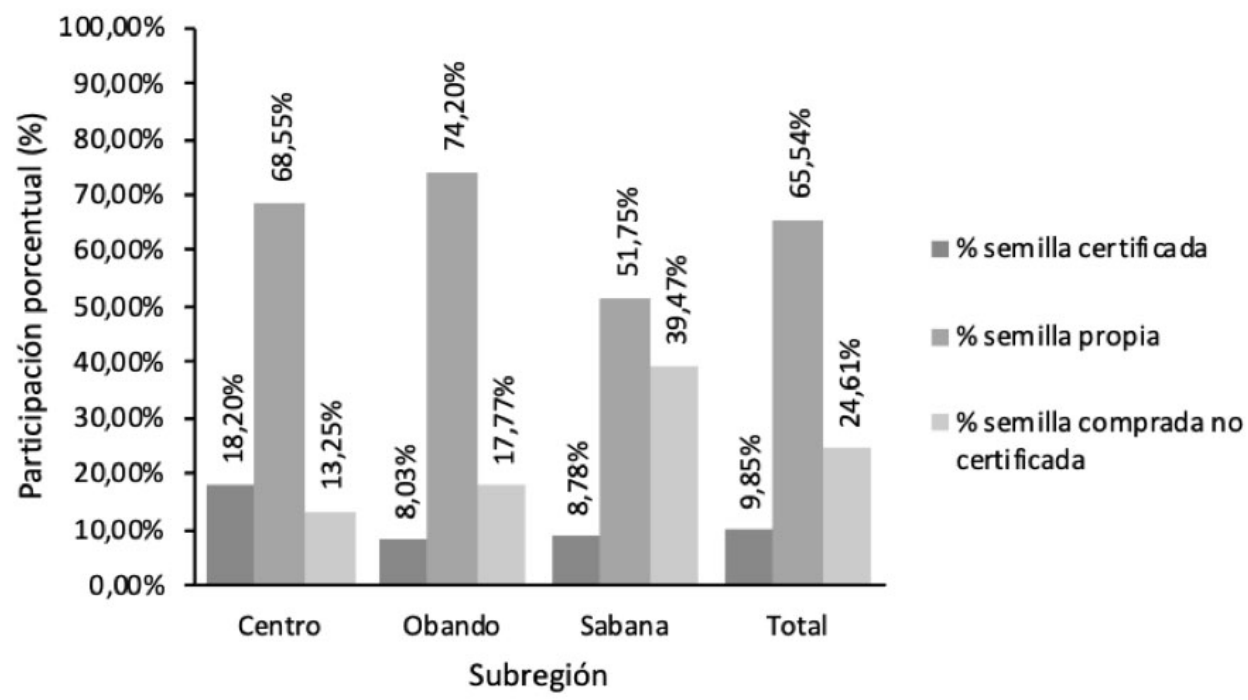

Figura 1. Participación porcentual de uso de semilla de papa (Solanum tuberosum L.), por subregión en el departamento de Nariño, Colombia. 2016.

Figure 1. Percentage share of potato seed use (Solanum tuberosum L.) by sub-region in the Nariño Department, Colombia. 2016.

Por subregiones, el mayor porcentaje de uso de semilla certificada se encuentra en la subregión Centro (18,2 $\%)$, correspondiente a los municipios de Pasto, Tangua y Yacuanquer. Es importante destacar que el uso de semillas compradas de tipo certificadas y descendientes de certificadas corresponde a productores de papa comercial con necesidades de 1,5 a $15 \mathrm{t}$. Para productores con capacidades de compra menores a 1,5 t, corresponde el porcentaje más alto de uso de semilla propia.

Para la subregión Exprovincia de Obando se encontró que el 74,2 \% correspondió a uso de semilla propia, el $17,7 \%$ a semillas descendientes de certificada y solo un 8,03\% utilizó semilla certificada, para un promedio del total de semilla usada de 3,3 t para esta subregión.

Para la subregión de la Sabana se encontró que el porcentaje de uso de semilla propia disminuyó en un 51,75 \% respecto a las otras subregiones, ya que el uso de semilla descendiente de certificada aumentó a un 39,47 \%; para el caso de la semilla certificada, su representatividad se estableció en un 8,78 \%. El promedio de uso de semilla para esta región fue de 4,49 t. 


\section{Zona potencial para la producción de semilla}

Las características biofísicas describen como áreas de producción aquellas que presentan suelos de textura franca, altitud entre 2800 y $3200 \mathrm{msnm}$ y temperatura de 8 a $15^{\circ} \mathrm{C}$. Además, es preciso tener presentes factores como el historial de producción agrícola del lote, el uso de suelos y las restricciones legales y ambientales.

Se encontró un área total aproximada de 34623 ha potenciales para la producción de semilla de papa, distribuidas principalmente en los municipios de Pasto, Sapuyes, Tangua, Cumbal, Guachucal y Puerres, que representan el $64,46 \%, 10,64 \%, 6,39 \%, 4,01 \%, 3,21 \%$ y 2,49 \%, respectivamente, del área total delimitada (Figura 2).

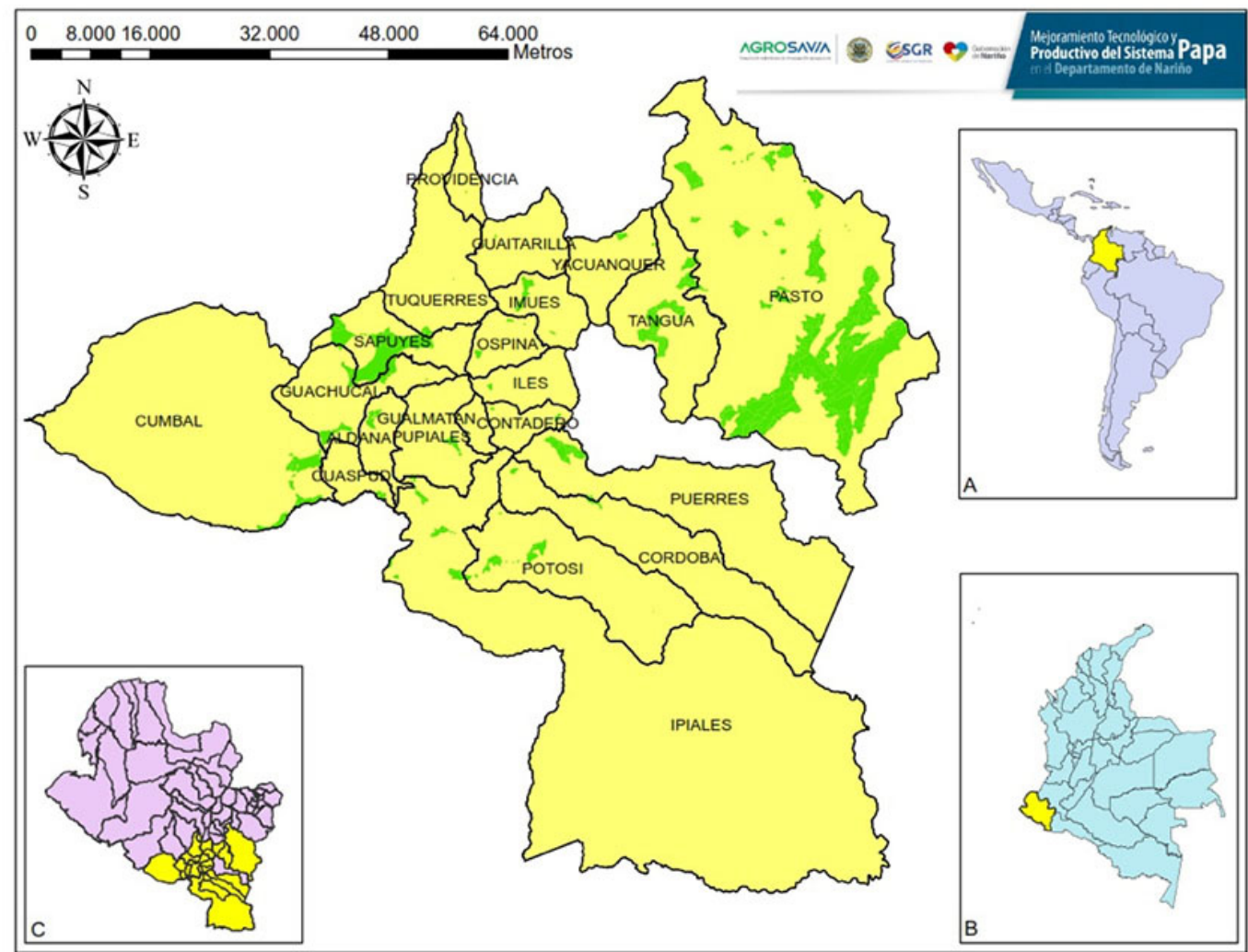

Figura 2. Zonas potenciales para la producción de semilla de papa (Solanum tuberosum L), en el departamento de Nariño, Colombia: a. Suramérica; b. Colombia; c. departamento de Nariño. 2016.

Figure 2. Potential areas for potato (Solanum tuberosum L) seed production in the Nariño department, Colombia: a. South America; b. Colombia; c. Nariño Department. 2016.

\section{Costos de producción de semilla de calidad}

En el Cuadro 1 se observan los rendimientos establecidos por categorías y tamaño de semilla, donde se determinaron, para cada una de estas, los siguientes costos: para la etapa de montaje de minitubérculos (2000 
Cuadro 1. Rendimientos establecidos por categorías y tamaños de semilla certificada de papa (Solanum tuberosum L.) para 0,1 ha (mini a básicas); 0,5 ha (básica a registrada) y 1 ha (registrada a certificada). Nariño, Colombia, 2018.

Table 1. Yields established by seed category and seed sizes of certified category of potato (Solanum tuberosum L.) for 0.1 (mini-tubers to basic seeds), 0.5 (basic to registered seeds), and 1 ha (registered to certified seeds). Nariño, Colombia, 2018.

\begin{tabular}{lccc}
\hline Tamaño de la papa / Categoría & $\begin{array}{c}\text { Mini a básicas (T)Área: } \\
\mathbf{0 , 1} \text { ha }\end{array}$ & $\begin{array}{c}\text { Básica a registrada (T)Área: } \\
\mathbf{0 , 5} \text { ha }\end{array}$ & $\begin{array}{c}\text { Registrada a certificada (T) } \\
\text { Área: } \mathbf{1} \text { ha }\end{array}$ \\
\hline $\begin{array}{l}\text { Primera / Muy grande } \\
(>90 \mathrm{~mm})\end{array}$ & 0,3 & 5 & 7,65 \\
Segunda / Mediana -pequeña & 1 & 15 & 24,6 \\
$(31-90 \mathrm{~mm})$ & & & \\
Tercera / Muy pequeña & & 1,5 & 2,3 \\
$(<30 \mathrm{~mm})$ & 0,2 & USD $\$ 4837$ & USD $\$ 8763$ \\
Costo de la etapa & USD $\$ 4245$ & & \\
\hline
\end{tabular}

unidades) a básicas en un área de $1000 \mathrm{~m}^{2}(0,1$ ha) se presentó un costo de USD 4245 , incluido el costo financiero (10\%); para básicas a registradas, con un área de $5000 \mathrm{~m}^{2}$ (0,5 ha), un costo de USD 4837; y finalmente, para registrada a certificada, en un área de $10000 \mathrm{~m}^{2}$ (1 ha), un valor de USD 8763. La principal carga económica fue la compra de insumos y servicios.

Con estas premisas, y teniendo en cuenta los cálculos de flujo de caja proyectados a siete años (cuatro ciclos formales de producción de semilla certificada) en escenarios de una a siete hectáreas, se concluyó que la carga económica no permite generar rendimientos financieros significativos en una sola hectárea ( $\overline{\mathrm{X}}: 5 \%$ ), ya que estos son inferiores a los esperados si el productor invirtiera los recursos en la producción de papa comercial y no de semilla, que equivale a un $18 \%$ en promedio. Por lo tanto, se sugiere como área óptima de cultivo tres hectáreas que producirían en promedio un $20 \%$ de rendimiento, donde el periodo promedio de recuperación de la inversión sería de tres ciclos productivos formales para semilla certificada, es decir, una media de seis años.

El Cuadro 2 indica que, si se tomaran en cuenta únicamente las tres hectáreas, el costo total por producir semilla certificada, desde la etapa de minitubérculos hasta certificada, sería USD 34 101,4, aunque los ingresos obtenidos

Cuadro 2. Ingresos netos generados en dólares para 0,1 ha (mini a básicas), 0,5 ha (básica a registrada) y 1 ha (registrada a certificada) de papa (Solanum tuberosum L), Nariño, Colombia. 2018.

Table 2. Net income in US dollars obtained for 0.1 ha (mini-tubers to basic seeds), 0.5 ha (basic to registered seeds), and 1 ha (registered to certified seeds) of potato (Solanum tuberosum L.). Nariño, Colombia. 2018.

\begin{tabular}{|c|c|c|c|}
\hline Tamaño de papa / Categoría & $\begin{array}{l}\text { Mini a básicas } \\
\quad(0,1 \text { ha })\end{array}$ & $\begin{array}{c}\text { Básica a registrada } \\
(0,5 \text { ha })\end{array}$ & $\begin{array}{l}\text { Registrada a certificada } \\
\text { (1 ha) }\end{array}$ \\
\hline Muy grandes & USD $\$ 66,6$ & USD $\$ 1110,4$ & USD $\$ 1699$ \\
\hline$(>90 \mathrm{~mm})$ & $\left(\mathrm{R}^{*}: 0,3 \mathrm{t} \mathrm{ha} \mathrm{a}^{-1}\right)$ & $\left(R^{*}: 5 t_{h a}^{-1}\right)$ & $\left(\mathrm{R}^{*}: 76,5 \mathrm{tha}^{-1}\right)$ \\
\hline Medina - pequeña & & USD $\$ 5286,3$ & USD $\$ 9983$ \\
\hline$(31-90 \mathrm{~mm})$ & & $\left(\mathrm{R}^{*}: 13 \mathrm{t} \mathrm{ha-1}\right)$ & $\left(\mathrm{R}^{*}: 24,6 \mathrm{tha}^{-1}\right)$ \\
\hline Muy pequeña & & US\$ 166,6 & USD $\$ 311$ \\
\hline$(<30 \mathrm{~mm})$ & & $\left(\mathrm{R}^{*}: 1,5 \mathrm{tha}^{-1}\right)$ & $\left(\mathrm{R}^{*}: 2,8 \mathrm{tha}{ }^{-1}\right)$ \\
\hline TOTAL & USD $\$ 66,6$ & USD $\$ 6563,3$ & USD \$11 993 \\
\hline
\end{tabular}

* R: rendimiento / * R: yield 
en la venta de la producción en cada etapa permitirían disminuir la carga financiera debido a su reinversión. Bajo el supuesto en que se diera la utilización completa de los recursos obtenidos por la venta de papa de primera (muy grande) (>90 mm), segunda (mediana y pequeña) $(31-90 \mathrm{~mm}$ ) y muy pequeña (coloquialmente conocida como richi o pollera) ( $<30 \mathrm{~mm}$ ), el costo real se establecería en USD 29098 y se presentarían utilidades de USD 6871.

La tasa interna de retorno para el área mínima de producción (3 ha) se estableció en un valor aproximado del $27 \%$.

\section{Demanda de semilla de papa en el departamento de Nariño}

Según cifras de la Red de Información y Comunicación de Agronet (Red de Información y Comunicación del Sector Agropecuario de Colombia) (Agronet, 2017), para 15924 ha sembradas por ciclo en Nariño, se necesitarían entre 23886 a 31847 t, y de ese total, el requerimiento en semilla certificada se establecería entre mínimo 717 t y máximo 955 t (3\%). Según el ICA (2018), las necesidades de semilla certificada estarían entre 1624 y 12166 t $(6,8 \%)$, y de acuerdo con la información de la encuesta efectuada para este trabajo, entre 2150 y 2866 t (9\%). Si al finalizar el 2017 el uso de semilla certificada se estableció alrededor del 6,8 \% y su demanda fue abastecida con semilla de Nariño y de la zona del Altiplano Cundiboyacense, la demanda potencial se establecería en los rangos de 908 a 1210 t, según datos de Fedepapa (2018), y de 525 a 701 t, según cifras generadas en el macroproyecto "Mejoramiento tecnológico y productivo del sistema papa en Nariño".

Con la producción que se esperaría obtener únicamente se estaría abasteciendo entre el $8 \%$ al $10 \%$, según Agronet (2017), entre un $3 \%$ al $5 \%$ de acuerdo con La Federación Colombiana de Productores de papa (Fedepapa, 2018), y alrededor de un $3 \%$ según la información de campo (encuesta); esto es, partiendo del hecho de que se cultiven como mínimo tres hectáreas para garantizar la rentabilidad del negocio.

Para el caso en que se cultivaran siete hectáreas, y con base en las proyecciones de Agronet (2017), se abastecería la demanda en mínimo un $18 \%$ y máximo un $24 \%$; usando las proyecciones de Fedepapa (2018), mínimo el $8 \%$ y máximo el $11 \%$, y si se usan los datos de la encuesta, mínimo el $6 \%$ y máximo un $8 \%$.

La demanda departamental promedio sin satisfacer si se cultivaran tres hectáreas sería del $90 \%$, y del $77 \%$ si se hiciera con siete. En cuanto a la oferta de productores a nivel nacional que se dedican al desarrollo de esta actividad, hay 73 grupos y únicamente cinco están en el departamento de Nariño (ICA, 2019).

\section{Dinámica de la comercialización de la producción de semilla}

En el flujograma de la dinámica de producción y comercialización de semilla para el departamento de Nariño (Figura 3), muestra la interacción de cada uno de los actores que involucra un proceso de sinergia no únicamente con los productores, sino con proveedores de minitubérculos y registradas, intermediarios, semilleristas y los mismos consumidores finales (productores pequeños, medianos y grandes), donde cada etapa implica la generación de estándares de calidad para el posicionamiento del negocio. En etapas de producción de básica y registrada el productor semillerista puede comercializar parte de la producción tanto de semilla como de papa de tamaño comercial, lo que le permitirá ingresos adicionales durante el proceso, una vez terminada la producción y comercialización de semillas certificadas, es necesario hacer una renovación de material con la obtención de mini tubérculos, o una etapa previa para darle dinamismo al ciclo. Todas las etapas son supervisadas por el ente regulador de Colombia (ICA). 


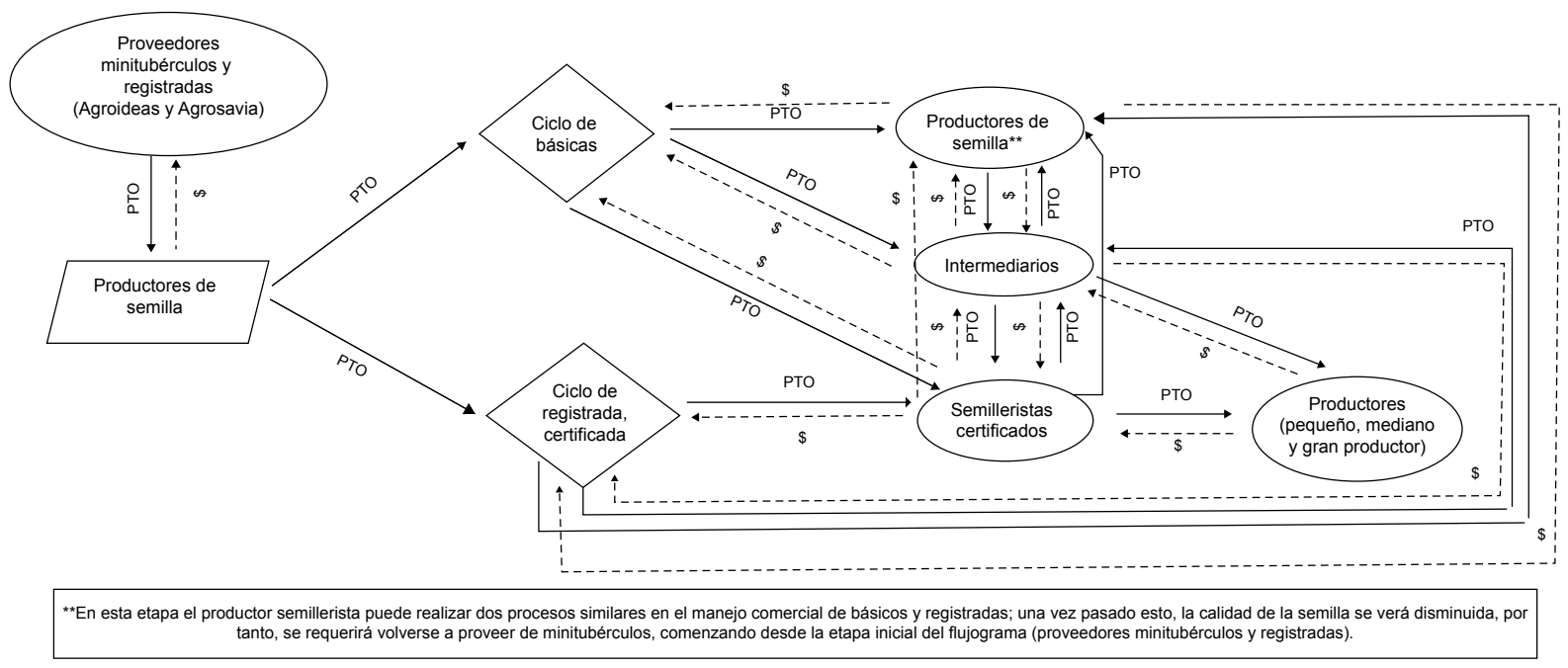

Figura 3. Flujograma del proceso de producción y comercialización de semilla de calidad de papa (Solanum tuberosum L). 2019.

Figure 3. Flowchart of the production and marketing process of quality potato seed (Solanum tuberosum L). 2019.

\section{Discusión}

Los resultados encontrados son acordes a lo registrado en países como Perú, Guatemala y Colombia, donde se cuenta con la existencia mayoritaria de una agricultura tradicional, caracterizada por el almacenamiento de semilla de la propia cosecha para iniciar la siguiente o la adquisición de esta por redes locales, familiares, comunales o pequeños distribuidores (Arenas-Calle et al., 2014).

Es evidente la presencia de productores con sistemas a gran escala, cuyos rendimientos económicos están asociados al uso de semillas de alta calidad y cultivos que se caracterizan por ser homogéneos, con altos índices de germinación, características fenotípicas deseables y un nivel considerable de resistencia a plagas y enfermedades (Bruíns, 2010; Teichert-Peske et al., 2010). Dichos insumos generalmente son producidos por empresas privadas, en programas de investigación y fitomejoramiento que requieren de una fuerte inversión financiera para su puesta en marcha. De acuerdo a los registros del Instituto Colombiano Agropecuario (ICA, 2018) para 2017, la oferta de productores nacionales que se dedicaban al desarrollo de esta actividad, contaba con un total de setenta organizaciones y únicamente cinco de ellas estaban en la región Andina del suroccidente de Colombia (Nariño): Agropecuaria Casanare, Carvajal Unigarro, Exa Agrícola E.U., Semilla de la Sabana y Semillas Nariño.

En lo concerniente a la descripción biofísica, esta estuvo acorde con el estudio realizado por la UPRA (2016), donde se identificaron las zonas con aptitud alta para la producción de papa comercial en temperaturas entre 8 y $16^{\circ} \mathrm{C}$, suelos de textura franca, franco-limosa, franco arenosa, limosa, arenosa, arenosa franca y orgánicos. Los semilleristas definieron la textura franco-arenosa como la más adecuada para la producción de semilla. Las zonas de producción de semillas deben reunir ciertos requisitos básicos relacionados con el aislamiento, la ausencia de factores limitantes como plagas y patógenos, las facilidades para la rotación y el acceso a caminos, entre otros. El conjunto de estos factores hace que una zona sea más o menos propicia para la producción de semilla (Hidalgo, 1999). 
Desde el componente económico, es necesario comprender que el diseño de costos de producción de papa dependerá en cierta medida de cada contexto territorial, a razón de que la estructura de costos en Colombia es muy heterogénea, debido a factores climatológicos y socioeconómicos, así como de la topografía, los sistemas de producción y los genotipos, entre otras condiciones que imposibilitan definir una estructura estándar (MADR, 2010), lo que conlleva a falta de bases actualizadas que permitan generar trazabilidad de los principales factores que se involucran en su diseño y medir el impacto de políticas que han sido orientadas al diseño de paquetes tecnológicos.

Bajo ese contexto, al cotejar la ratio de la TIR del área mínima de producción (3 ha) con cálculos efectuados en investigaciones de tecnologías de aeroponía (Mateus-Rodriguez et al., 2013), presentó una disminución de doce unidades porcentuales. No obstante, el escenario exhibe una oportunidad si se compara con la venta de papa comercial cuyo precio presenta gran variabilidad en el transcurso del tiempo. Un ejemplo de ello es lo sucedido en 2016 y 2017 (Fedepapa, 2018), cuando los precios de compra se definieron en USD 14,23 y 10,12, respectivamente y generaron utilidades por la producción de una hectárea de papa de variedad Diacol Capiro para el año 2016 de USD 1054 y para 2017 de USD -1704. En contraste, estos remanentes siguen siendo inferiores a los del negocio de semilla, cuyas utilidades en una hectárea, si se difieren los ingresos netos obtenidos de las tres hectáreas, corresponderían a USD 3228,27, es decir, la rentabilidad del negocio de producción de semilla de calidad en una hectárea sería inferior en 0,1\% si se compara con la producción de papa comercial (TIR: $18 \%$ ); por lo que es recomendable como área optima de producción, tres hectáreas para generar un panorama económico favorable (TIR: $27 \%$ ), aunque su implementación requiere de un buen manejo financiero, especialmente durante el primer año.

El desarrollo de este tipo de negocios requerirá del fortalecimiento de capacidades a nivel individual, institucional y un entorno normativo favorable (Neate y Guei, 2011) en cuanto a planificación y gestión de la producción, almacenamiento de semilla, comercialización, contabilidad e incluso ejecución de operaciones agrícolas especializadas que permitan su viabilidad, especialmente para pequeñas organizaciones de productores que se encaminan hacia estos emprendimientos. Es así como el apoyo técnico es crucial en las primeras etapas del desarrollo de la empresa de semilla. De otra parte, el generar material de buena calidad, con pureza varietal a precios competitivos, además de las relaciones confiables que se vinculen en el desarrollo de la actividad, se establecerán en factores diferenciadores (Salinas et al., 2011). Esta hipótesis puede ser apreciada en los resultados obtenidos en el presente estudio (Figura 3) para el sistema formal de producción de semilla en Colombia.

El proceso del sistema informal también juega un papel importante en el desarrollo de la industria semillera del país al constituirse en un abastecedor a pequeña escala para la agricultura; no obstante, en Colombia, a diferencia de países como Brasil, Guatemala y Perú, no se cuenta con estudios y estadísticas actualizadas sobre la participación informal de semilla que permitan generar políticas de articulación con los pequeños agricultores (Arenas-Calle et al., 2014).

En general, los precios de semilla certificada son más estables y no varían como los precios de la papa comercial. La volatilidad de los precios se atribuye a factores predominantes como la estacionalidad de la producción, el carácter perecedero del tubérculo y la inestabilidad del precio y la demanda, entre otros factores que han generado vulnerabilidad en el sector (Barrientos et al., 2014). Esta hipótesis ha sido respaldada a través de modelos económicos en los cuales se determinó que entre 2004 y 2009 los precios de variedades como la Diacol Capiro, tuvieron un ciclo de treinta meses, lo cual indica que al final de ese lapso la papa alcanzó su precio más bajo o su precio más alto, dependiendo de las condiciones del mercado (Mosquera, 2011). Es en estas circunstancias el productor se constituye en el agente con menor poder de decisión en la definición del precio como estrategia para el incremento de sus ingresos, debido a que las condiciones deficientes e inadecuadas del sistema de comercialización han conllevado a que se vea obligado a vender su producción de inmediato a los intermediarios, y estos últimos agentes son quienes definen el precio y designan las reglas de juego a su conveniencia (Manjarrés-Rojas y Molinade-Paredes, 2007). 
Desde hace unos treinta años en los países desarrollados, las diversas condiciones que afectan los ingresos en la producción de papa comercial, donde se han implementado acciones por parte de los entes gubernamentales para mitigar dichos cambios, vienen siendo analizados; sin embargo, sus labores siguen sin ser contundentes (Horton, 1988), debido a la falta de planificación del cultivo y de información que tienen los pequeños y medianos productores, lo cual no permite generar condiciones de mercado justo (Martínez-Covaleda et al., 2005; Gómez, 2008).

\section{Conclusiones}

Para el año 2016 la producción de papa comercial en su gran mayoría fue originada a partir de semilla propia, mientras el área sembrada con semilla certificada fue la de menor participación, lo que indica que existe una amplia demanda de semilla para implementar cultivos comerciales, pero se presenta una baja oferta o acceso limitado a semilla de calidad.

La carga económica no permitió generar rendimientos significativos en una sola hectárea (6\%) de producción de semilla de papa, debido a que fueron inferiores al rendimiento de la producción de papa comercial (18\%).

El área óptima de cultivo, fue de tres hectáreas que producirían en promedio un $20 \%$ de rendimiento, una tasa interna de retorno (TIR) del $28 \%$ y un abastecimiento del mercado en torno al $3 \%$, según la información de campo.

En la delimitación de áreas potenciales para la producción de semilla, las mayores áreas con características biofísicas adecuadas se encuentran en los Municipios de Pasto, Tangua y Sapuyes.

\section{Conflicto de intereses}

Los autores han cumplido las normas de publicación y no generan conflicto de interés en la presente investigación.

\section{Agradecimientos}

Se agradece el apoyo financiero al Fondo Ciencia Tecnología e Innovación del Sistema General de Regalías (SGR), al Departamento Académico de Ciencia, Tecnología e Innovación (Colciencias), al ejecutor del proyecto: Gobernación de Nariño, y a los operadores: Corporación Colombiana de Investigación Agropecuaria (Agrosavia) y Universidad de Nariño (Udenar). A los Investigadores Luis Fernando Gómez y Pedro Uribe por su gestión y administración del proyecto.

\section{Literatura citada}

Agronet (Red de Información y Comunicación del Sector Agropecuario de Colombia). 2017. Estadísticas agropecuarias 2016/2017. https://www.agronet.gov.co/estadistica/Paginas/home.aspx (consultado 15 oct. 2018).

Arenas-Calle, W.C., C.I. Cardozo-Conde, y M. Baena. 2014. Análisis de los sistemas de semillas en países de América Latina. Acta Agron. 64:239-245. doi:10.15446/acag.v64n3.43985

BANREP (Banco de la república de Colombia). 2019. Catálogo de estadísticas. BANREP, COL. https://www.banrep.gov.co/es/ catalogo-estadisticas-disponibles\#captacion-semanales (consultado 28 feb. 2020).

Barrientos, J.C., C. Rondón, y S.E. Melo. 2014. Comportamiento de precios de las variedades de papa Parda Pastusa y Diacol Capiro en Colombia (1995-2011). Rev. Colomb. Cienc. Hortic. 8:272-286. doi:10.17584/rcch.2014v8i2.3220 
Borba, N. 2008. La papa, un alimento básico: posibles impactos frente a la introducción de papa transgénica. RAP-AL, Montevideo, URY.

Bruíns, M. 2010. El aporte del mejoramiento vegetal para la agricultura. Seed News, BRA. https://seednews.com.br/ artigos/1578-el-aporte-del-mejoramiento-vegetal-para-la-agricultura-edicao-janeiro-2010(consultado 17 oct. 2018).

Cámara de Comercio de Pasto. 2017. Sondeo de percepción económica tercer trimestre, 2017. Departamento de planeación y competitividad regional. Cámara de Comercio de Pasto, COL. https://www.ccpasto.org.co/wp-content/uploads/2018/01/ Sondeo-de-Percepcion-III-Trimestre-Final-1.pdf (consultado 31 oct. 2017).

Cubbage, F., R. Davis, y G. Frey. 2011. Guía para la evaluación económica y financiera de proyectos forestales comunitarios en México. Documento de Trabajo Forestal Latinoamericano nº 2. PROFOR, MEX.

DANE (Departamento Administrativo Nacional de Estadística). 2018a. Sistema de información de precios SIPSA, 20122017. DANE, COL. https://www.dane.gov.co/index.php/estadisticas-por-tema/agropecuario/sistema-de-informacion-deprecios-sipsa (consultado 18 ene. 2018).

DANE (Departamento Administrativo Nacional de Estadística). 2018b. Índices de precios al consumidor. DANE, COL. https:// www.dane.gov.co/index.php/estadisticas-por-tema/precios-y-costos/indice-de-precios-al-consumidor-ipc/ipc-historico (consultado 18 ene. 2018).

Devaux, A., M. Ordinola, A. Hibon, y R. Flores. 2010. El sector papa en la región andina: diagnóstico y elementos para una visión estratégica (Bolivia, Ecuador y Perú). Centro Internacional de la Papa, Lima, PER.

Díaz-Bravo, L., U. Torruco-García, M. Martínez-Hernández, y M. Varela-Ruiz. 2013. La entrevista, recurso flexible y dinámico. Inv. Ed. Med. 2(7):162-167. doi:10.1016/S2007-5057(13)72706-6

Esprella, R., P. Flores, y J. García. 2012. Guía práctica para producir nuestra semilla de papa de calidad: Guía para agricultores/ agricultoras y técnicos. Centro Internacional de la Papa, La Paz, BOL.

Ezeta, F.N. 2001. Producción de semilla de papa en Latinoamérica. Rev. Latinoam. Papa 12:1-14.

Fedepapa (Federación Colombiana de Productores de Papa). 2018. Histórico de precios departamento de Nariño, Colombia, 2012-2017. Fedepapa, COL. https://fedepapa.com/boletin-historico-narino/ (consultado 20 ene. 2018).

Gittinger, J. 1983. Análisis económico de proyectos agrícolas. 2a ed. Editorial Tecno S.A., Madrid, ESP.

Gobernación de Nariño. 2013. Cultivos transitorios, papa. Consolidado agropecuario de Nariño 2013, San Juan de Pasto, COL.

Gómez, M. 2008. La papa, su comercialización y el caso especial frente a la comunidad de Pasquilla. Tesis Esp., Escuela Superior de Administración Pública, Bogotá, COL.

Hidalgo, O. 1999. Conceptos básicos sobre la producción de semilla de papa y sus instituciones. En: O. Hidalgo, editor, Producción de tubérculo semilla de papa. Centro Internacional de la Papa, Lima, PER. p. 1-22.

Horton, D. 1988. Las papas en los países en desarrollo. Rev. Latinoam. Papa 1(1):9-17.

ICA (Instituto Colombiano Agropecuario). 2015. Resolución n 3168 del 7 de septiembre. ICA, COL. https://www.ica.gov.co/ getattachment/4e8c3698-8fcb-4e42-80e7-a6c7acde9bf8/2015R3168.aspx (consultado 20 feb. 2019).

ICA (Instituto Colombiano Agropecuario). 2018. Informe de gestión 2017. ICA, COL. http://www.ica.gov.co/Multimedia/swf/ RevistaVirtual/2015/Informe_Gestion_ICA_2017.pdf (consultado 24 feb. 2019).

ICA (Instituto Colombiano Agropecuario). 2019. Registros D.T. semillas, certificados de semillas. ICA, COL. https://www. ica.gov.co/getdoc/08d0b08f-f704-4e0f-bfb2-14f861fb5215/certificacion-de-semillas .aspx (consultado 25 oct. de 2019). 
MADR (Ministerio de Agricultura y Desarrollo Rural) 2010. Acuerdo de competitividad de la cadena agroalimentaria de la papa en Colombia. MADR, COL. https://sioc.minagricultura.gov.co/DocumentosContexto/P008-NUEVO\%20ACUERDO\%20 DE\%20COMPETITIVIDAD\%20PAPA.pdf\#search=cadena\%20de\%20la\%20papa (consultado 25 feb. 2019).

MADR (Ministerio de Agricultura y Desarrollo Rural). 2018. Cadena de papa: indicadores e instrumentos. MADR, COL. https:// sioc.minagricultura.gov.co/Papa/Documentos/2018-12-30\%20Cifras\%20Sectoriales.pdf (consultado 25 feb. 2019).

Manjarrés-Rojas, E., y O. Molina-de-Paredes. 2007. Proceso de cálculo del costo de producción del rubro papa, municipio Rangel del estado Mérida. Visión Gerencial 1:73-81.

Martínez-Covaleda, H.J., C.F. Espinal, N. Pinzón-Ruíz, y C.A. Barrios-Urrutia. 2005. La cadena de la papa en Colombia: una mirada global de su estructura y dinámica, 1991-2005. Ministerio de Agricultura y Desarrollo Rural, Bogotá, COL.

Mateus-Rodriguez, J.R., S. Hann, J.L. Andrade-Piedra, L. Maldonado, G. Hareau, I. Barker, C. Chuquillanqui, V. Otazú, R. Frisancho, C. Bastos, A.S. Pereira, C.A. Medeiros, F. Montesdeoca, and J. Benítez. 2013. Technical and economic analysis of aeroponics and other systems for potato mini-tuber production in Latin America. Am. J. Potato Res. 90:357368. doi:10.1007/s12230-013-9312-5

Mete, M.R. 2014. Valor actual neto y la tasa de retorno: su utilidad como herramienta para el análisis y evaluación de proyectos de inversión. Fides et Ratio 7(7):67-85.

Morales, A., y J. Morales. 2014. Planeación financiera. Grupo Editorial Patria, México D.F., MEX.

Mosquera, F. 2011. Diseño de un modelo de planificación de cultivos para mejorar el desempeño de pequeños y medianos productores de papa, en el municipio de Pasto, departamento de Nariño. Tesis MSc., Universidad de la Sabana, Chía, COL.

Murray, S., y S. Larry. 2009. Estadística. 4ta ed. Mc. Graw-Hill, México, D.F., MEX.

Neate, P.J.H., y R.G. Guei. 2011. Promoción del crecimiento y desarrollo de empresas de semillas de pequeños agricultores en cultivos para la seguridad alimentaria. Mejores prácticas y opciones para la toma de decisiones. FAO, Roma, ITA.

Ñústez, C.E. 2011. Variedades colombianas de papa. Universidad Nacional de Colombia, Bogotá, COL.

Puente-Montañez, G.A. 2011. Formulación y evaluación de proyectos agropecuarios. Ecoe Ediciones, Bogotá, COL.

Salinas, M., L. Tamblay, y C. Loyola. 2011. Resultados y lecciones en producción y comercialización de la papa. Fundación para la Innovación Agraria, Ministerio de Agricultura, Santiago, CHL.

Teichert-Peske, S., A.C. Souza-de-Alburquerque-Barros, y L.O. Braga-Schuch. 2010. Beneficios y obtención de semillas de alta calidad. Seed News, BRA. https://seednews.com.br/artigos/1874-beneficios-y-obtencion-de-semillas-de-alta-calidadedicao-setembro-2010 (consultado 25 oct. 2018).

UPRA (Unidad de Planificación Rural Agropecuaria). 2016. Cultivo comercial de papa: identificación de zonas aptas en Colombia, a escala 1:100.000. UPRA, COL. UPRA, COL. https://www.upra.gov.co/documents/10184/13821/ Zonificaci\%C3\%B3n_papa (consultado 25 oct. 2018). 